International Journal of Pure and Applied Mathematics

Volume 87 No. 2 2013, 301-305

ISSN: 1311-8080 (printed version); ISSN: 1314-3395 (on-line version)

url: http://www.ijpam.eu

doi: http://dx.doi.org/10.12732/ijpam.v87i2.11

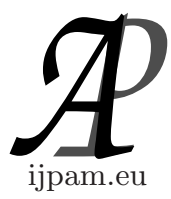

\title{
MONTE CARLO METHOD BASED ON INEXACT NEWTON TO THE SVIP WITH CONSTRAINT CONDITIONS
}

\author{
Shuang Chen ${ }^{1 \S}$, Zunquan Xia ${ }^{2}$, Deshui $\mathrm{Yu}^{3}$, Fanyun Meng ${ }^{4}$ \\ $1,2,3,4$ Ganjingzi District \\ Dalian, Liaoning Province, Linggong 2, P.R. CHINA
}

\begin{abstract}
In this paper we apply the Monte Carlo method based on inexact Newton to solving a class of stochastic variational inequality problems (SVIPs). Locally convergence results of the proposed methods are proved under appropriate conditions. It show that the conditions we impose are satisfied and that the solutions, efficiently generated by proposed the Monte Carlo inexact Newton-procedure, have desirable properties.
\end{abstract}

AMS Subject Classification: 90C15, 65C05

Key Words: stochastic variational inequality problem, Newton approximation

\section{Introduction}

Variational inequality problem (VIP for short) has a number of important applications in operations research, engineering problems and economic equilibrium problems. While many practical problems not only involve deterministic data, there are some important instances where problem data contains some uncertainties and consequently stochastic variational inequality problem (SVIP) models are proposed to reflect the uncertainties.

Received: April 21, 2013

(c) 2013 Academic Publications, Ltd.

$\S$ Correspondence author url: www.acadpubl.eu 
Finding $x \in X$ satisfying

$$
(y-x)^{T} E[f(x, \xi(\theta))] \geq 0, y \in X,
$$

where $\xi(\theta)$ is a random variate defined on a probability space $(\Omega, F, P), f(\cdot, \xi)$ : $R^{n} \longrightarrow R^{n}$ is locally Lipschitz and the feasible set $X \subseteq R^{n}$ is described by some equality and inequality constraints: $X=\{x \mid h(x)=0, l(x) \geq 0\}$, where the functions $h$ and $l$ are assumed to be affine.

In the past few years, a lot of work [1], [3], [4], [5] have been done for the stochastic variational inequality problem. In [1] they investigate exact Newton method for solving SVIP. However, in practical implementations it may be computationally very expensive to solve the Newton equation (3.2) of [1] exactly. This is true also if the model $A(x, \cdot)$ is linear (as in the smooth case) but very large. It is then useful to consider solving the Newton equation inexactly.

In this paper, we consider Monte Carlo method which based on inexact Newton-method for the stochastic variational inequality problem. It turns out that all the properties they have established for the exact Newton method remain valid if we solve the Newton equation in a suitable accurate way as stipulated by and. These two conditions provide a unified framework for inexact nonsmooth Newton schemes and we give the proof of locally convergence.

The rest of the paper is organized as follows. In the next Section, we introduce some basic definitions and transform SVIP to stochastic nonsmooth equation. In Section 3, we use SA methods that are based on inexact Newtonmethod for solving SVIP and give the proof of the convergence.

\section{Monte Carlo Method Based on Inexact Newton-Method}

We often try to solve the corresponding nonlinear system of equations instead of $(1)$.

$$
G(s):=\left(\begin{array}{c}
E[f(x, \xi)]+\nabla h(x) y+\nabla l(x) z \\
h(x) \\
\phi(l(x), z)
\end{array}\right)=0 .
$$

Now we consider the following Robbins-Monro type stochastic approximation (iterative) scheme for solving the SVIP:

$$
s^{k+1}=s^{k}+d^{k}+w^{k},
$$

where $d^{k}$ is the true value of Newton direction at $s^{k}, w^{k}$ is a stochastic error.

To explain how iterative scheme (2) works, let us consider $G(s)=E\left[g\left(s, \xi^{k}\right)\right]$ where $\xi^{k}$ is a stochastic sample of random variate. Then we can find $w^{k}$ such 
that $g\left(s, \xi^{k}\right)=G(s)+w^{k}$. Then we do not need to know the probability distribution of $\xi$ for approximating $G(s)$.

Next we introduce main algorithm of this paper.

\section{Algorithm 3.1.}

Step 1: Set $\mathrm{k}=0, s^{0} \in R^{n}$ and $\varepsilon>0$.

Step 2: If $G\left(s^{k}\right)=0$, stop.

Step 3: Select an element $A\left(s^{k}, \cdot\right)$ in $\mathcal{A}\left(s^{k}\right)$ and find a direction $d^{k}$ in $\mathrm{B}(0, \varepsilon)$ such that

$$
G\left(s^{k}\right)+A\left(s^{k}, d^{k}\right)=r^{k},
$$

where $r^{k}$ is a vector satisfying

$$
\left\|r^{k}\right\| \leq \eta_{k}\left\|G\left(x^{k}\right)\right\|
$$

and $\mathcal{A}$ is a Newton approximation scheme [2] for $G$ at $\bar{s}$.

Step 4: $\operatorname{Set} s^{k+1} \equiv s^{k}+d^{k}+w^{k}$, where $w^{k}$ is a stochastic error. Set $k=k+1$, go to Step 2 .

\section{Convergence Analysis}

The main theoretical results are list below:

Lemma 1. Let $G: \Omega \subset R^{n} \rightarrow R^{n}$ with $\Omega$ open, be a locally Lipschitz function in a neighborhood of $s^{*} \in \Omega R^{n}$ satisfying $G\left(s^{*}\right)=0$. Assume that $G$ admit a nonsingular Newton approximation $\mathcal{A}$ at $s^{*}$, For every $\varepsilon \in\left(0, \varepsilon_{\mathbf{A}}\right]$ and every $\bar{\eta}>0$, a neighborhood $\mathbf{B}\left(s^{*}, \delta\right)$ of $s^{*}$ exists such that for every scalar $\eta_{k} \in(0, \bar{\eta}]$, every vector $s_{k} \in \mathbf{B}\left(s^{*}, \delta\right)$ and every vector $r^{k}$ satisfying (4) the equation (3) has a unique solution $d^{k}$ in $\mathrm{B}(0, \varepsilon)$.

Theorem 2. Let $G: \Omega \subseteq R^{n} \rightarrow R^{n}$ with $\Omega$ open, be a locally Lipshitz function in a neighborhood of $s^{*} \in \Omega$ satisfying $G\left(s^{*}\right)=0$. Assume that $G$ admits a nonsingular Newton approximation $\mathcal{A}$ at $s^{*}$. There exist a positive number $\bar{\eta}>0$, such that if $\eta_{k} \leq \bar{\eta}$ for every $k$ then for every $\varepsilon \in\left(0, \varepsilon_{\mathbf{A}}\right]$, a neighborhood $\boldsymbol{B}\left(s^{*}, \delta\right)$ of $s^{*}$ exists such that if $s^{0}$ belongs to $\boldsymbol{B}\left(s^{*}, \delta\right)$, the inexact Newton method is well defined and $E\left[w^{k} \mid F^{k}\right]=0 ; E\left[\left\|w^{k}\right\|^{2} \mid F^{k}<\infty\right]$ then the Newton Algorithm generates a sequence $\left\{s^{k}\right\}$ that converges to $s^{*}$.

Proof. Let $\epsilon$ be any fixed constant in the interval $\left(0, \epsilon_{\mathrm{A}}\right]$. we first note that by Definition 7.2.2 of [2] can find a positive $\delta$ such that for every $s \in B\left(s^{*}, \delta\right)$ 
and for every $A(x, \cdot) \in \mathcal{A}(x)$ we can write

$$
\left\|G(x)+A\left(s, s^{*}-s\right)\right\|=\left\|G(s)+A\left(s, s^{*}-s\right)-G\left(s^{*}\right)\right\| \leq\left(2 L_{\mathrm{A}}\right)^{-1}\left\|s-s^{*}\right\|,
$$

where $L_{\mathrm{A}}$ is the Lipschitz constant in condition(c)of the Definition 7.2.2 of [2].

Because of $G$ is Lipschitz continue, we can choose $\delta>0$ to be less than $\min (2 \epsilon, \epsilon / L)$ and also such that for all $x \in B\left(s^{*}, \delta\right)$,

$$
\|G(s)\|=\left\|G(s)-G\left(s^{*}\right)\right\| \leq L\left\|s-s^{*}\right\| \leq L \delta<\epsilon,
$$

where $L$ is a Lipschitz constant of $G$ around $s^{*}$. Due to Lemma 1, we know (3) has a unique solution.

$$
\begin{aligned}
E\left[\left\|s^{k+1}-s^{*}\right\|^{2} \mid F_{k}\right]= & E\left[\left\|s^{k+1}+d^{k}+w^{k}-s^{*}\right\|^{2} \mid F_{k}\right] \\
= & E\left[\left\|s^{k}+A^{-1}\left(s^{k},-G\left(s^{k}\right)+r^{k}\right)+w^{k}-s^{*}\right\|^{2} \mid F_{k}\right] \\
= & E\left[\|-A^{-1}\left(s^{k}, A\left(s^{k}, s^{*}-s^{k}\right)\right)+A^{-1}\left(s^{k},-G\left(s^{k}\right)+r^{k}\right)\right. \\
& \left.+w^{k} \|^{2} \mid F_{k}\right] \\
\leq & L_{\mathrm{A}}^{2}\left\|G\left(s^{k}\right)-r^{k}+A\left(s^{k}, s^{*}-s^{k}\right)\right\|^{2}+E\left[w^{2} \mid F_{k}\right] \\
\leq & L_{\mathrm{A}}^{2}\left[\left\|s^{k}-s^{*}\right\| \triangle\left(\left\|s^{k}-s^{*}\right\|\right)+\left\|r^{k}\right\|\right]+E\left[w^{k^{2}} \mid F_{k}\right] \\
\leq & L_{\mathrm{A}}^{2}\left[\left\|s^{k}-s^{*}\right\| \triangle\left(\left\|s^{k}-s^{*}\right\|\right)+\eta^{k}\left\|G\left(s^{k}\right)\right\|\right] \\
& +E\left[w^{k^{2}} \mid F_{k}\right] \\
\leq & L_{\mathrm{A}}^{2}\left[\left\|s^{k}-s^{*}\right\| \triangle\left(\left\|s^{k}-s^{*}\right\|\right)+\bar{\eta} L\left\|s^{k}-s^{*}\right\|\right] \\
& +E\left[w^{k^{2}} \mid F_{k}\right] .
\end{aligned}
$$

Make $\bar{\eta}$ and $\delta$ Sufficiently small, then there exists a count $\kappa$ between 0 and 1 such that

$$
E\left[\left\|s^{k+1}-s^{*}\right\|^{2} \mid F_{k}\right] \leq\left\|s^{*}-s^{k}\right\|^{2}-\kappa\left\|s^{*}-s^{k}\right\|^{2}+E\left[w^{k^{2}} \mid F_{k}\right]
$$

For $\left\|s^{k+1}-s^{*}\right\|$ convergent almost sure and $\sum \kappa\left\|s^{*}-s^{k}\right\|^{2}<\infty$ is satisfy almost sure. For the Lemma 3.1 in [1] we have that $\left\|s^{*}-s^{k}\right\|$ convergence to 0 .

\section{References}

[1] S. Chen., L.P. Pang, F.F. Guo, Z.Q. Xia, Stochastic methods based on Newton method to the stochastic variational inequality problem with constraint conditions, Mathematical and Computer Modelling, 55 (2012), 779784 .

[2] F. Facchinei, J.S. Pang, Finite-Dimensional Variational Inequalities and Complementarity Problems, Springer, New York, 2003. 
[3] J. Filar, K. Vrieze, Competitive Markov Decision Processes, SpringerVerlag, 1997.

[4] G. Gürkan, A.Y. Özge, S.M. Robinson, Sample-path solution of stochastic variational inequalities, Mathematical Programming, 84 (1999), 313-333.

[5] H.Y. Jiang, H.F. Xu. Stochastic approximation approaches to the stochastic variational inequality problem, IEEE Transactions on Automatic Contro, 53 (2008), 1462-1475. 
